\title{
Research and Application of Active Noise Control System in Vehicle
}

\author{
Fuchen Ding a), Mingjiang Wang ${ }^{\mathrm{b})}$ and Xing $\mathrm{Wu}^{\mathrm{c}}$ \\ School of Electronic and Information Engineering, Harbin Institute of Technology, Shenzhen 518000, China. \\ a)1191915012@qq.com \\ b) Corresponding author: mjwang@hit.edu.cn \\ c)hit_wx@126.com
}

\begin{abstract}
With the improvement of living standard, riding comfort has become an important indicator of choosing a car, but the noise in the car is the key factor affecting the ride comfort. The traditional passive noise reduction technology has a good inhibitory effect on high frequency noise, but the effect of low frequency noise is not ideal, and the active noise control system solves this problem well. This article first analyzes the characteristics and components of automobile noise, this paper introduces the principle of active noise control system, introduces the structure of the feed-forward and feedback structure and mixed structure of active noise control system structure characteristics and performance, and analyzes its feasibility for automobile noise suppression. The minimum mean square (LMS) algorithm and the filtering X minimum mean square (FX-LMS) algorithm are introduced. Based on DSP development platform, the identification algorithm of the secondary acoustic channel and the ANC control algorithm are completed in the one-dimensional sound field. The noise source includes narrowband noise and broadband noise. Set up a single channel in the test vehicle. The experiment results show that the system has certain control effect on the broadband noise.
\end{abstract}

Keywords: Active Noise Control, interior noise, Least Mean Square, Secondary path.

\section{INTRODUCTION}

In the mid-1930s, a patent published by German physicist Paul Lueg proposed a method for controlling the noise of pipes. It is generally recognized as the beginning of research on active noise reduction [1]. In 1953, U.S. RCA researchers Harry Olson and Everet May followed up with Leug. After they perfected and improved the basic principle of active noise reduction technology, the electronic muffler device invented by classical control technology was first proposed [2]. After that, they applied the device to the back of the car, and the active noise reduction technology in the automobile or aircraft field has been on the human history stage [3].

Nowadays, active noise reduction technology is applied in more scenes. At the same time, the adaptive filtering algorithm has gained the favor of more researchers [4]. Real life has been widely popularized, such as active noise cancelling headphones, aircraft passenger cabin de-noising, automobile active noise reduction technology and so on[5]. But even the active noise reduction technology based on adaptive filter to obtain a large number of practical applications, but because of the degeneration of the higher requirements for fashion, and random noise in broad frequency range and other factors, the adaptive active noise reduction technology is still not mature enough, distance and stable, easy to implement and faster processing speed applications need more and more people focus and resources devoted to research and promotion [6]. 


\section{THE BASIC PRINCIPLE OF ACTIVE NOISE CONTROL}

\section{Adaptive Filtering Algorithm}

The key to the noise reduction performance of noise control system is the output $y(n)$ of the adaptive filter and the degree of coherence of the primary noise. The higher the coherence of the two signals, the smaller the residual noise and the better the noise reduction effect [7]. On the contrary, the effect of noise reduction is not good, and even the noise may be enhanced. So the selection of adaptive filter and adaptive algorithm is very important. Generally speaking, the selection of adaptive filter has two kinds of FIR filter IIR filter, the order number of IIR filter is low, but the existence of zero pole may cause the instability of the system. The order of FIR filter is relatively high, which will improve the computation, but it is more stable and convenient than IIR filter, so we choose the horizontal filter FIR here. The adaptive filtering algorithm adjusts the coefficients of the filter by minimizing the square value of $e(n)$, while the minimum mean square algorithm is simple, and most of the adaptive filtering algorithms are the cost functions. Therefore, the minimum mean square criterion is selected as the cost function to adjust the filter coefficients. This paper analyzes the adaptive filtering algorithm based on the feedforward active noise control system, and the algorithm framework is shown in figure 1:

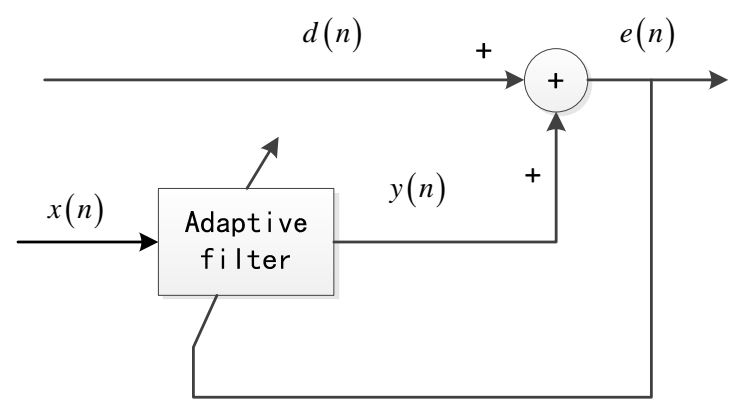

FIGURE 1. Algorithm Framework of Forward Active Noise Control System.

Where $d(n)$ as the original expectations of noise signal, $x(n)$ reference microphone to pick up the reference signal, $y(n)$ for secondary signal adaptive controller output, $e(n)$ for the desired signal $d(n)$ and the control system output secondary signal $y(n)$ after the superposition of residual noise. The input reference signal $x(n)$ and weight coefficient $\mathrm{w}(\mathrm{n})$ of the adaptive horizontal filter at the NTH time are defined as follows, where $\mathrm{n}$ is the number of the filter weight coefficient or the number of the filter tapped.

$$
\begin{gathered}
X(n)=[x(n), x(n-1), x(n-2), \ldots \ldots, x(n-N+1)]^{T} \\
W(n)=[w(n), w(n-1), w(n-2), \ldots \ldots, w(n-N+1)]^{T}
\end{gathered}
$$

The output secondary signal of adaptive filter is:

$$
y(n)=X^{T}(n) * W(n)
$$

Noise residuals picked up by the error microphone:

$$
e(n)=d(n)-y(n)
$$

According to the steepest descent method, the iterative updating formula of the filter coefficients is as follows: 


$$
w(n+1)=w(n)+\mu e(n) * x(n)
$$

In the decline, it is a real thing to control the filter of every iteration of the filter and its influence on the control system's collection of speed and stability. If you are on the right side, you will be able to collect yourself. If value is too large, the system is not easy to convergence or even divergence.

\section{Feedforward Active Noise Control System}

Feedforward active noise control system is by putting the reference microphone to pick up the reference signal through a adaptive filter, get a the same as the original noise signal amplitude phase instead of secondary signal, through the secondary speaker, after playing with the original noise superposition is a residual error microphone get noise, provided to the adaptive filter is used to change the filter weight, achieve the goal of adaptive noise control. The reference signal is $\mathrm{x}(n)$, and the output signal of the secondary source isy $(n)$. The error signal after the superposition of the secondary source and the primary noise ise $(n)$. The schematic diagram is as follows:

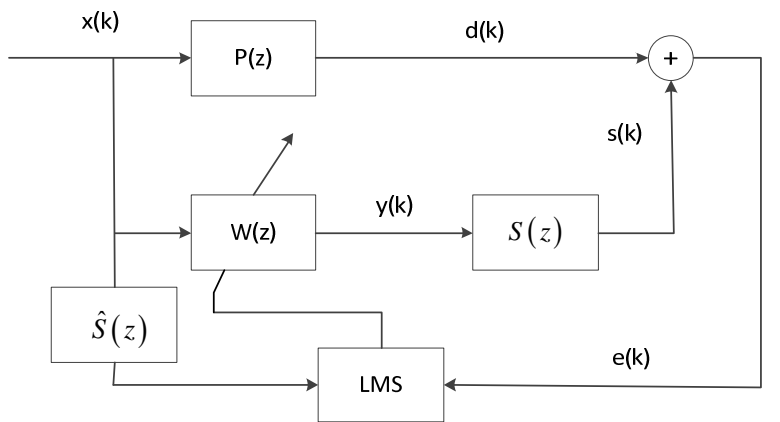

FIGURE 2. FXLMS Adaptive Algorithm Block Diagram.

By the cloth in microphone near the noise measured signal as reference signalx $(n)$, the input to the adaptive controller, and then by the controller of the reference signal filtering processing, such as the output signal y $(n)$ after meet certain characteristics of the output to the secondary sound source. Error of the cloth in the secondary sound source near microphone, receives the primary source and secondary source after the superposition of acoustic signal and error signal via feedback adaptive controller to adjust the weights and correction coefficient of filter, the system gradually achieves the best state.

\section{SIMULATION RESULTS}

For feedforward active noise systems is the actual noise experiment, using the car engine, simulation system for noise reduction.

Figure 3 for the result of the experiment, can be observed that the car engine noise has been suppressed, from large to small amplitude final convergence, control signal with the original noise is also on the way, in the end.

The purpose of this project is to apply the active noise control system to the vehicle, and we simulate the interior space simulation to experiment with the $3 \mathrm{~d}$ enclosed space. The length and width of the space are $100 \mathrm{~cm}, 70 \mathrm{~cm}$ and $70 \mathrm{~cm}$ respectively, and each wall is a rigid wall. To facilitate the observation of the position of the internal device and reduce the external influence, we use plexiglass to build the enclosed space. The left and right sides have two circular holes with a radius of $1 \mathrm{~cm}$, which is used to arrange power lines. In order to visually observe the sound pressure distribution, a 3D mapping method is used to draw a closed spatial sound field distribution diagram.

We divide the length and width of the three sides by 9,6 and 6, and the enclosed space consists of 324 measuring points. When playing primary noise, the sound pressure measured by decibel meter is shown in Figure 4. 

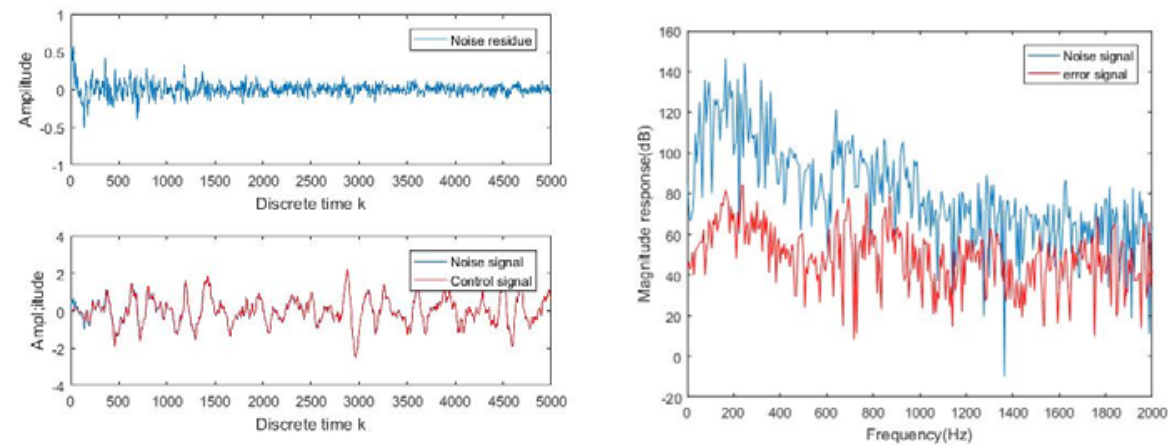

FIGURE 3. Simulation Experiment of Car Engine.

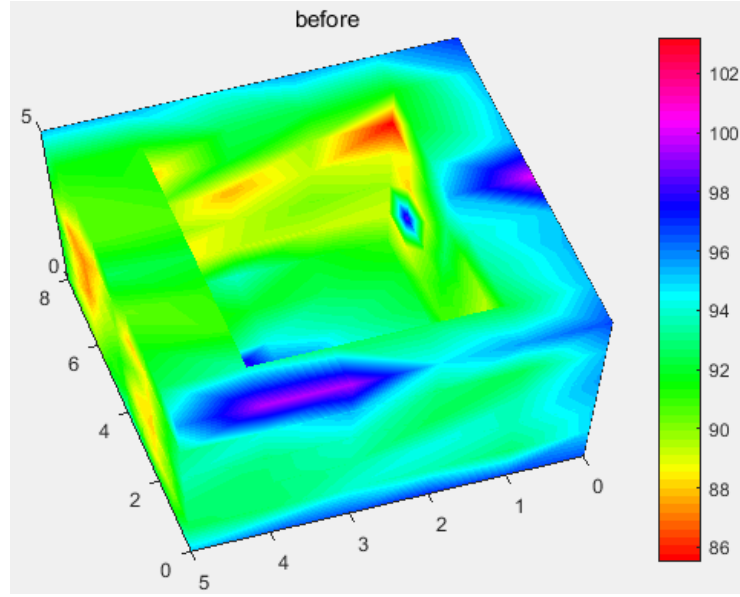

FIGURE 4. Closed Space Offsets the Internal Sound Pressure Level Distribution.

After the secondary noise is played, the primary and secondary noise are superimposed on each other in the whole enclosed space, and in some regions, there is an increase in some areas. The sound field distribution after cancellation is shown in figure 5 .

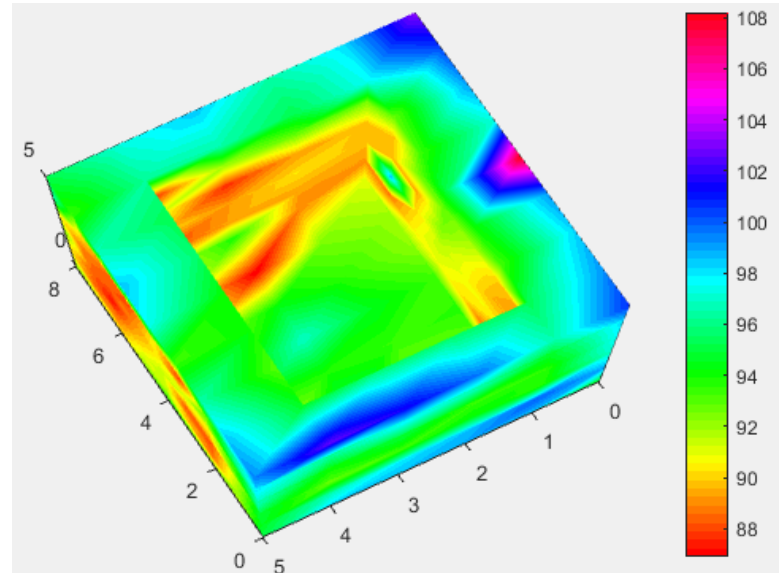

FIGURE 5. The Internal Sound Pressure Level Distribution After the Closed Space Offsets. 
After the offset of the distribution of sound pressure in as you can see, in the unity of the two speakers plane has the obvious effect of noise reduction, in a central location, at the bottom of the error we see the original blue area near the microphone has become a green area, noise reduction in roughly about 5-9 dB, and bottom position in some other corner, also appeared different degree of color change, there are less noise reduce. In other places, the edges and corners, the center of the side, have different degrees of enhancement.

\section{CONCLUSION}

In this paper, the basic principle of active noise control system is described in detail and the corresponding simulation results are given. Compared with LMS algorithm, FXLMS algorithm has the advantages of good noise reduction, fast convergence speed and strong robustness. However, the noise composition in the vehicle is more complex, and the FXLMS algorithm has a limited effect on its noise reduction, which is further improved to improve the noise reduction effect.

\section{REFERENCES}

1. Leug P. Process of silencing sound oscillation: Germany, 655508[P]. 1933:5-11.

2. Olson H F, May E G. Electronic sound absorber [J]. The Journal of the Acoustical Society of America, 1953, 25(6): 1130-1136.

3. Kuo S M, Morgan D R. Active Noise Control: A Tutorial Review [J]. Proceedings of the IEEE, 1999, 87(6): 943-973.

4. Chiou Y H, Chang C Y, Kuo S M. Active Noise Control Systems for Periodic Noise Signals[C]//Proceedings of SICE Annual Conference. IEEE, 2012: 1783-1786.

5. Nakrani N, Patel N. Feed-Forward and Feedback Active Noise Control System Using FxLMS Algorithm for Narrowband and Broadband Noise[C]//International Conference on Communication Systems and Network Technologies. IEEE, 2012: 577-580.

6. S. C. Chan and Y. Chu, "Performance analysis and design of FxLMS algorithm in broadband ANC system with online secondary-path modeling," IEEE Trans. Audio, Speech, Lang. Process., vol. 20, no. 3, pp.982-993, Mar. 2012.

7. Kean Chen. Active Noise Control [M]. Beijing: Defense industry press, 2003:1-3. 